\title{
Atypical Renal Cysts: Is MRI a Compulsory Examination in 2020 before Therapeutic Management?
}

\author{
Idriss Ziani1,2, Ahmed Ibrahimi1,2, Hamza Dergamoun1,2, Omar Belouki1,2, Hachem El Sayegh ${ }^{1,2}$, \\ Lounis Benslimane ${ }^{1,2}$, Yassine Nouini ${ }^{1,2}$
}

${ }^{1}$ Urological Surgery Department “A”, IBN Sina University Hospital, CHU RABAT, Rabat, Morocco

${ }^{2}$ RABAT Faculty of Medicine, Mohammed V University, Rabat, Morocco

Email: idrissziani20@gmail.com

How to cite this paper: Ziani, I., Ibrahimi, A., Dergamoun, H., Belouki, O., El Sayegh, H., Benslimane, L. and Nouini, Y. (2020) Atypical Renal Cysts: Is MRI a Compulsory Examination in 2020 before Therapeutic Management? Open Journal of Urology, 10, 253-262.

https://doi.org/10.4236/oju.2020.1010030

Received: September 10, 2020

Accepted: October 11, 2020

Published: October 14, 2020

Copyright $\odot 2020$ by author(s) and Scientific Research Publishing Inc. This work is licensed under the Creative Commons Attribution International License (CC BY 4.0).

http://creativecommons.org/licenses/by/4.0/

\begin{abstract}
Introduction: Kidney cancer represents $2 \%$ to $3 \%$ of malignant tumors, with an incidence of 5.8/100,000 in Western countries, a very specific subgroup of renal tumors that are cystic tumors. In fact, almost $50 \%$ of the population over 50 has a renal cyst. Materials and Methods: The objective of this study is to establish a correlation between the preoperative Bosniak score and the postoperative anatomopathological analysis in order to answer this question, the use of contrast ultrasound or MRI should be systematically performed before an atypical renal cyst? This is a retrospective study of 50 patients treated for renal cysts between January 2010 and March 2016. All patients have an abdominopelvic ultrasound and an abdominal scanner. Cysts have been classified according to the Bosniak classification. We treated our patients based on the radiological examination urocsanner and we classified our patients according to the classification of Bosniak, and uro MRI was performed in cases of atypical cysts or diagnostic doubt before considering a therapeutic attituderding to the classification of Bosniak. Results: In our work, the Uro MRI was sharper for atypical cysts (type IIF and Type III cyst) with sensitivity greater than that of the scanner but with a specificity similar to that of the scanner. And therefore be a key examination in the atypical kidney cysts. Conclusions: URO MRI is currently in 2020 a key examination of atypical bonsiak IIf and III cysts before management of the therapeutic load.
\end{abstract}

\section{Keywords}

Atypical Cyst, BosniaK, Cystic Tumors, URO MRI 


\section{Introduction}

Kidney cancer represents $2 \%$ to $3 \%$ of malignant tumors [1], with an incidence of 5.8/100,000 in Western countries [2]. In 2012 in France, its incidence was estimated at more than 11,500 new cases with a male/female ratio of 2.5 [3]. In Europe, almost 84,400 new cases have been diagnosed and more than 34,700 deaths linked to kidney cancer have been recorded [4]. The incidence of these cancers has greatly increased due to the development of imaging tests such as $\mathrm{CT}$, magnetic resonance imaging (MRI) or ultrasound which allow to discover these tumors at an asymptomatic stage, of smaller pruning and at an earlier stage [5]. A very specific subgroup of kidney tumors is cystic tumor. In fact, almost $50 \%$ of the population over the age of 50 has a renal cyst.

Cystic tumors are represented by atypical cystic lesions or secondary cystic kidney tumors. The objective is to establish the criteria mentioned for tumor proliferation requiring histological confirmation and excision. Cystic kidney images are recognized according to the classification proposed in 1986 by the Bosniakon the basis of scan criteria [6] [7] then currently renewed in 2019. The main modifications integrate MRI into this new classification. [8]

The purpose of this study is to establish a correlation between the preoperative Bosnian score and the postoperative anatomopathological analysis in order to answer this question, MRI should be performed in front of an atypical cyst or a cystic mass of the kidney?

\section{Materials and Methods}

This is a retrospective study of 50 patients treated for renal cysts between January 2010 and March 2016. All the patients underwent an abdominopelvic ultrasound and an abdominal computed tomography. The cysts were classified according to the Bosniak classification.

The data collected were analyzed retrospectively and include: age, sex, circumstances of discovery, radiological data, Bosniak classification and histology of the cyst after excision. As well as a follow-up of each patient is on an average follow-up of 30 months.

We calculated the sensitivity and specificity of the radiological explorations in this case of CT in the detection of cystic kidney cancers by comparing the imaging data and the final histological results. We excluded from the study patients under conservative treatment for a type I or II bosiank cyst

The data in the literature are collected through a systematic review of the literature on PubMed and on Science Direct using the terms: "cystic renal tumors, complex renal cysts, Bosniak classification, Bosniak IIF category”. Prospective and retrospective studies in adults, in French and in English, as well as reviews of the literature on the subject were reviewed.

\section{Results}

Our series includes 50 patients supported in the urological surgery department 
"A" of CHU RABAT for renal cyst over a period of 5 years between January 2010 and March 2016, the series includes, 35 men and 15 women of middle age 42 years (29 to 73 years), The circumstances of the discovery were variable: Accidental discovery: 25 cases (50\% of cases) during an ultrasound for reasons other than urological, Low back pain: 20 cases, Hematuria: 5 cases.

The initial radiological assessment includes an abdominal ultrasound, the latter being systematically completed in the event of renal injury by a scanner in order to classify cystic lesions according to the Bosniak classification (Table 1).

Among symptomatic cysts, $60 \%$ were at least one BosniaK IIF while $40 \%$ of symptomatic cysts were not suspect (type I and II) (Figures 1(a)-(d), Figure 2).

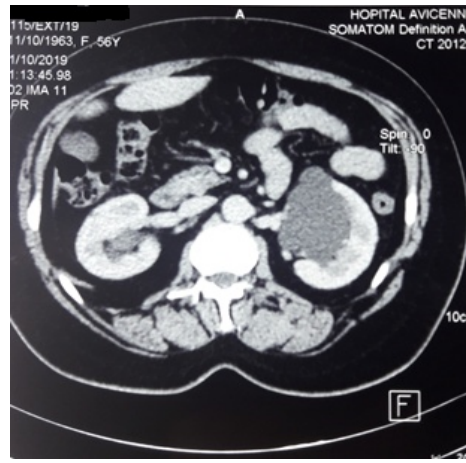

(a)

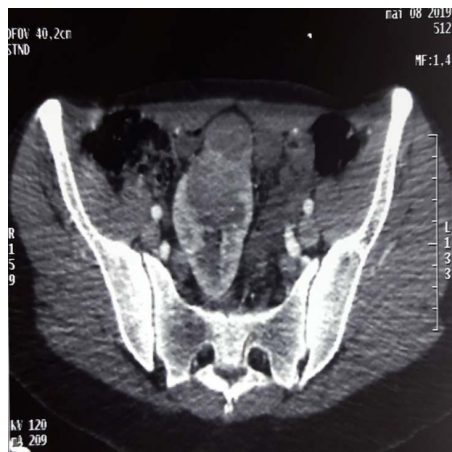

(c)

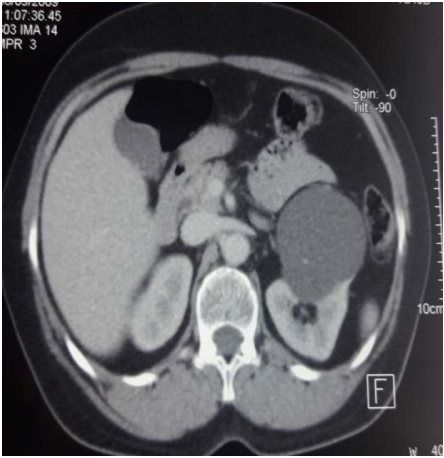

(b)

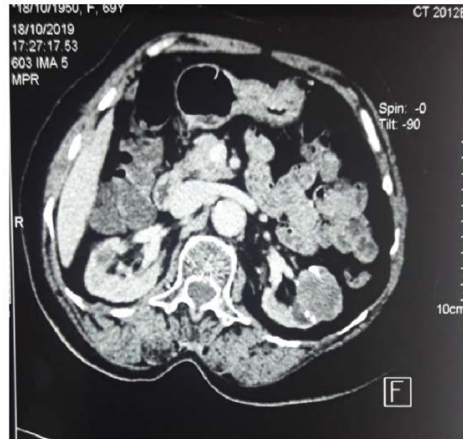

(d)

Figure 1. (a) CT image: typical parapyelic cyst of the Bosniak I kidney; (b) CT image: renal cyst classified as Bosniak II; (c) CT image: atypical cyst on a pelvic ectopic Bosniak kidney calasse IIF; (d) CT image: Bosniak V calsated kidney cyst.

Table 1. Results of the radiological evaluation according to the CT scan classification of the Bosniak.

\begin{tabular}{cc}
\hline $\begin{array}{c}\text { Modified classification of cystic } \\
\text { lesions of the kidney according to the Bosniak }\end{array}$ & Number of patient \\
\hline Type I Simple cyst & 17 \\
Type II Atypical cyst & 10 \\
Type IIF & 4 \\
Type III Suspect cyst & 10 \\
Type IV Cystic cancer & 9 \\
\hline
\end{tabular}




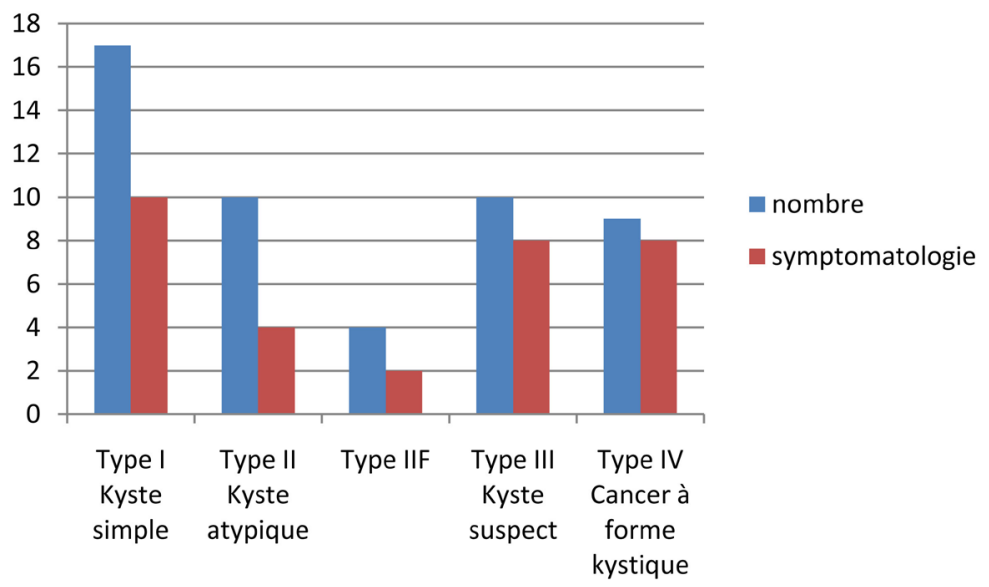

Figure 2. Clinical symptomatology based on radiological data.

For the therapeutic management, a total of 35 patients treated as such, patients suspected of Bosniak cysts III and IV all benefited from a nephrectomy respecting the rules of cancer:

13 total nephrectomies and 6 partial nephrectomies, among the 13 total nephrectomies 9 by laparoscopy, 4 by open route. The 6 partial nephrectomies were performed by open route.

For non-suspect cysts (type I or II): 11 treated surgically and 3 patients with a Bosniak cyst 1 with occasional minimal symptoms, benefit from sclerotherapy for puncture of the cyst.

Finally two IIF cysts were treated by lower polar partial nephrectomy due to the rapid increase in volume during monitoring.

No operative or postoperative complications were noted (Figure 3).

We treated our patients on the basis of the urocsanner radiological examination and on the classification of our patients according to the classification of Bosniak, the MRI was carried out in case of atypical cysts or diagnostic doubt before considering a therapeutic attitude.

We calculated the sensitivity and specificity of this CT classification by comparing the results of the preoperative scanner with the anatomopathological results of the operating parts.

The anatomopathological characteristic according to the classification of Bosniak is illustrated in the attached table (Figure 4).

As illustrated in table $\mathrm{n}=2$, of the 19 patients operated on for a suspected cancerous cyst (Bosnian III and IV), 14 presented tumor lesions (sensitivity $75 \%$ ). These were localized (pT1 and pT2) and low-grade cystic tumors (Furhman I and II). For Bosnian VI cysts, the sensitivity was $100 \%$. Of the 11 patients operated on for cyst II or I, no tumor lesion was detected (specificity of 100\%) and for the two patients operated on for cyst IIF, only one patient presented with cancer (specificity and sensitivity of 50\%) (Table 2). Uro MRI was performed in 4 patients with IIF cysts, allowed the reclassification of a single patient into cyst III and whose histological analysis was in favor of a low-grade carcinomatous 


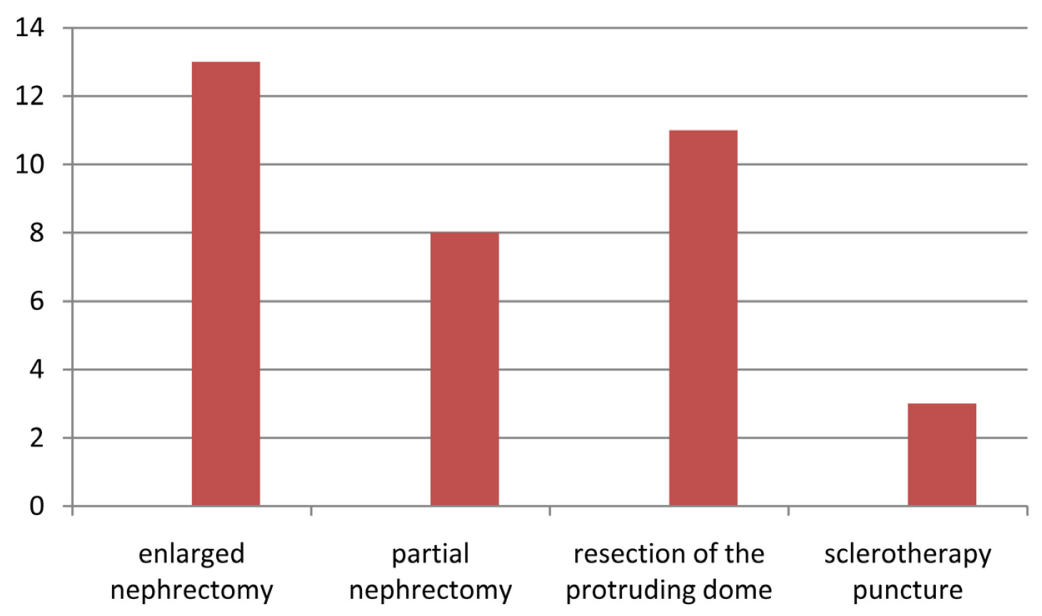

Figure 3. Distribution of patients according to therapeutic modalities.

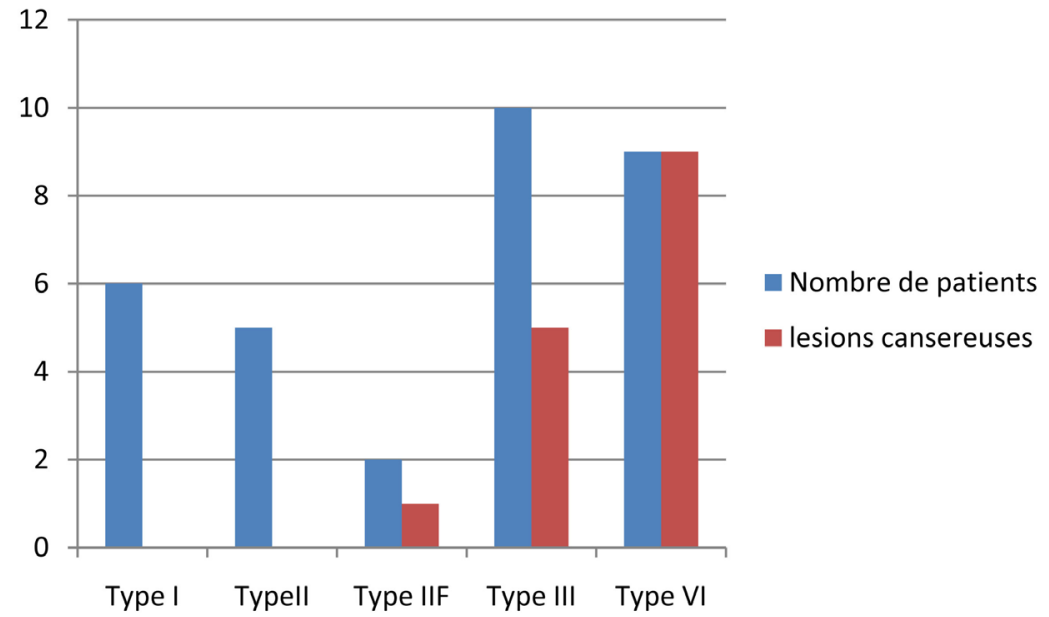

Figure 4. Anatomopathological characteristic according to the classification of the Bosniak.

Table 2. Specificity and sensitivity of CT.

\begin{tabular}{ccc}
\hline type of cyst & number of patients & with cancerous lesions \\
\hline Type I & 8 & 0 detected (specificity of $100 \%)$ \\
Type II & 6 & O detected (specificity of $100 \%)$ \\
Type IIF & 2 & 1 detected (specificity and sensitivity of $50 \%)$ \\
Type III & 10 & 5 detected specificity and sensitivity of $50 \%)$ \\
Type VI & 9 & 9 detected (100\% sensitivity) \\
\hline
\end{tabular}

lesion, a uro a MRI was also performed in 5 patients with type III cyst whose computed tomography was questionable also allowing reclassification in two type VI patients and the histological analysis was in favor of the malignant tumor, finally In 5 cases, an MRI was requested in the presence of a sacnnographic doubt (type II and type VI cyst) and whose results were similar to those of the scanner and there was no reclassification (Table 3 ). 
Table 3. Reclassification rate after MRI.

\begin{tabular}{cccc}
\hline $\begin{array}{c}\text { type } \\
\text { of cyst }\end{array}$ & $\begin{array}{c}\text { number of } \\
\text { patients }\end{array}$ & with cancerous lesions (CT) & $\begin{array}{c}\text { Reclassification rate } \\
\text { (MRI)/under } \\
\text { staging by CT }\end{array}$ \\
\hline Type I & 6 & 0 detected (specificity of 100\%) & - \\
Type II & 5 & O detected (specificity of 100\%) & $0 \%$ \\
Type IIF & 2 & 1 detected (specificity and sensitivity of 50\%) & $50 \%$ \\
Type III & 10 & 5 detected specificity and sensitivity of 50\%) & $40 \%$ \\
Type VI & 9 & 9 detected (100\% sensitivity) & $0 \%$ \\
\hline
\end{tabular}

In our work, Uro MRI was sharper for atypical cysts (type IIF and Type III cyst) with a sensitivity greater than that of the scanner but with a specificity similar to that of the scanner and therefore having been a key examination in the atypical kidney cysts.

Regarding follow-up, no late or recurrent complications were noted in patients treated surgically or by sclerotherapy with a follow-up of 30 months.

\section{Discussion}

The incidence of kidney cancer has increased considerably thanks to the development of imaging tests such as CT (CT), magnetic resonance imaging (MRI) or ultrasound, which allow these tumors to be discovered at an asymptomatic stage, smaller in size and at an early stage [5]. The same goes for a very specific subgroup of kidney tumors, namely cystic tumors.

For over 30 years, the Bosniak classification of cystic kidney masses has been proposed for the first time [9]. This classification based on computed tomography was introduced in 1986 and initially divided into cystic renal masses in one of the four classes after exclusion of infectious, inflammatory and vascular etiologies [9].

It is not used for lesions of urothelial (hydrocalice), vascular (aneurysm) or infectious (abscess) origin. This classification allows to separate typical cysts (types I and II), atypical cysts (type IIF and type III) and carcinomatous cysts (type IV). This classification is based on several criteria including the density, the presence of partitions and their morphology, the appearance of their walls, the presence of vegetation or the contrast product of these elements. Depending on the type of cyst, there is a correlation with the potential for malignancy [10] which guides therapeutic management. In case of diagnostic doubt, the contrast ultrasound [11] and MRI [12] report can be useful in determining the nature of atypical cysts. Indeed, some studies have obtained better diagnostic accuracy with contrast ultrasound compared to CT (90\% vs 74\%) [11].

Although initially described from the CT description of renal cysts [6], the Bosniak classification is currently adapted to MRI with at least equal histopathological correlation [12]. Israel et al. report a benefit of MRI on the scanner on certain lesions, thanks to better spatial resolution allowing better detection of the 
number and thickness of the partitions as well as their contrast enhancement [13]. The predictors of neoplasia are similar to those described for CT. Recent literature has focused on the contribution of diffusion MRI, based on the movement of water molecules at the cellular level, in the detection of suspicious cysts. The cellularity of tumors and healthy tissue being different, the MRI signal seems to be modified. The advantage is that it does not require gadolinium injection. If the first results are encouraging, this technique cannot by itself replace traditional MRI and morphological imaging is still necessary [12]. (Figure 3)

Histologically, cystic cancers represent $5 \%$ to $7 \%$ of kidney tumors [14] [15]. Among cystic tumors, the most common histology is clear cell cystic carcinoma (63\%), followed by papillary carcinoma (25\%). They are generally of good prognosis because they are not very aggressive and of low grade. Reese et al. report $79 \%$ of pT 1 tumors and $73 \%$ of Fuhrman grade 1 or 2 tumors in the case of neoplastic cystic tumors [14].

\section{Historically aggressive management of kidney masses suspected of being carcinoma}

The renal cell (RCC) [15] [16], led to the resection of numerous benign masses and indolent cancers, without benefiting the patients. This may be particularly true for cystic kidney cancers, which are less likely to be malignant and, which are cancerous, are more likely to be indolent and have a better prognosis [17] [18] [19].

Since cystic kidney masses are often benign, there is a need to improve their imaging-based characterization so that the cancers that need to be treated are identified and surgery for benign diagnoses is avoided. It begins with a critical assessment of the Bosniak classification. In the diagnosis of cystic CRC, it is essential to balance the risks of active therapy (i.e. loss of nephron, treatment-related morbidity, costs) with those of active surveillance [15].

The proposed update of the Bosnian classification remains a malignancy prediction system, not a complete management algorithm. Patient factors such as age, co-morbidities, life expectancy, preferences and risk tolerance must all be taken into account in a treatment plan and adapted from simple recommendations for each Bosnian class. However, in general, ignoring the class I and II masses, observing the class IIF masses and considering the resection or removal of the class IV masses will probably remain the pillars of the management of the Bosniak classification, 2019 version. Observation is always recommended for Bosnian IIF cystic masses because about $10 \%$ of these masses see progress in imaging, and those with a progression of about $85 \%$ are likely to be malignant [15] [17]. It is not clear whether Class III masses offer the best for observation or treatment in the general population (i.e. patients without comorbidities or with a limited life expectancy).

Regarding our series, the scanner had a sensitivity and specificity close to $100 \%$ for Bosniak II and IV cysts whereas for atypical kytes (IIF, III), MRI was much more effective.

MRI was performed in 14 patients, 4 patients with IIF cyst, 5 patients with 
type III cyst, MRI allowed reclassification to a more suspect stage in $50 \%$ of cases, and $40 \%$ of patients with IIF cyst and III respectively and whose histological analysis was all clever in other patients with cysts II and VI no reclassification thanks to MRI.

Therefore better specificity and sensitivity are close to $100 \%$ in atypical cysts (IIF and III).

In our series, all of the cancerous lesions were clear cell cystic carcinomas with a low grade of Fuhrman.

\section{Conclusions}

The natural history of cysts and cystic kidney tumors is now known and their optimal management is well identified. It is based on their morphological description according to the Bosniak classification, which can be performed by CT, MRI or contrast ultrasound.

Bosniak cysts I and II are considered mild and do not require treatment only if they are symptomatic and have no potential for malignancy, while types III and IV require surgical excision in accordance with cancer rules.

Types IIF can benefit from surveillance by imaging after being sure to eliminate under-starification, with a risk of progression of $15 \%$ to $30 \%$, of which the patient must be informed, and any morphological modification during surveillance must lead to cancer surgery excision.

In our study, MRI showed superiority over CT in atypical IIF and III cysts with significant reclassification rates.

In the other simple cysts (type I and II) and carcinogenic cysts (type V), there was no significant difference compared to CT.

\section{Conflicts of Interest}

The authors declare that they have no competing interests.

\section{References}

[1] European Network of Cancer Registries (2001) Eurocim Version 4.0. European Incidence Database V2.3, 730 Entity Dictionary.

[2] Jemal, A., Bray, F., Center, M.M., Ferlay, J., Ward, E. and Forman, D. (2011) Global Cancer Statistics. CA: A Cancer Journal for Clinicians, 61, 69-90. https://doi.org/10.3322/caac.20107

[3] Rébillard, X., Grosclaude, P., Leone, N., Velten, M., Coureau, G., Villers, A., et al. (2013) [Incidence and Mortality of Urological Cancers in 2012 in France]. Progrès en Urologie, 23, S57-S65. https://doi.org/10.1016/S1166-7087(13)70047-2

[4] Ferlay, J., Steliarova-Foucher, E., Lortet-Tieulent, J., Rosso, S., Coe-Bergh, J.W.W., Comber, H., et al. (2013) Cancer Incidence and Mortality Patterns in Europe: Estimates for 40 Countries in 2012. European Journal of Cancer, 49, 1374-1403.

[5] Patard, J.-J., Rodriguez, A., Rioux-Leclercq, N., Guillé, F. and Lobel, B. (2002) Prognostic Significance of the Mode of Detection in Renal Tumours. BJU International, 90, 358-363. https://doi.org/10.1046/j.1464-410X.2002.02910.x

[6] Bosniak, M.A. (1986) The Current Radiological Approach to Renal Cysts. Radiolo- 
gy, 158, 1-10. https://doi.org/10.1148/radiology.158.1.3510019

[7] Bosniak, M.A. (1997) The Use of the Bosniak Classification System for Renal Cysts and Cystic Tumors. Journal of Urology, 157, 1852-1853.

https://doi.org/10.1016/S0022-5347(01)64883-3

[8] Silverman, S.G., Pedrosa, I., Ellis, J.H., Hindman, N.M., Schieda, N., Smith, A.D., Remer, E.M., Shinagare, A.B., Curci, N.E., Raman, S.S., Wells, S.A., Kaffenberger, S.D., Wang, Z.J., Chandarana, H. and Davenport, M.S. (2019) Bosniak Classification of Cystic Renal Masses, Version 2019: An Update Proposal and Needs Assessment. Radiology, 292, 475-488. https://doi.org/10.1148/radiol.2019182646

[9] Graumann, O., Osther, S.S. and Osther, P.J.S. (2011) Characterization of Complex Renal Cysts: A Critical Evaluation of the Bosniak Classification. Scandinavian Journal of Urology and Nephrology, 45, 84-90.

https://doi.org/10.3109/00365599.2010.533695

[10] Park, B.K., Kim, B., Kim, S.H., Ko, K., Lee, H.M. and Choi, H.Y. (2007) Assessment of Cystic Renal Masses Based on Bosniak Classification: Comparison of CT and Contrast-Enhanced US. European Journal of Radiology, 61, 310-314. https://doi.org/10.1016/j.ejrad.2006.10.004

[11] Ellimoottil, C., Greco, K.A., Hart, S., Patel, T., Sheikh, M.M., Turk T.M.T., et al. (2014) New Modalities for Evaluation and Surveillance of Complex Renal Cysts. Journal of Urology, 192, 1604-1611. https://doi.org/10.1016/j.juro.2014.07.099

[12] Israel, G.M., Hindman, N. and Bosniak, M.A. (2004) Evaluation of Cystic Renal Masses: Comparison of CT and MR Imaging by Using the Bosniak Classification System. Radiology, 231, 365-371. https://doi.org/10.1148/radiol.2312031025

[13] Reese, A.C., Johnson, P.T., Gorin, M.A., Pierorazio, P.M., Allaf, M.E., Fishman, E.K., et al. (2014) Pathological Characteristics and Radiographic Correlates of Complex Renal Cysts. Urologic Oncology, 32, 1010-1016. https://doi.org/10.1016/j.urolonc.2014.02.022

[14] Cooperberg, M.R., Mallin, K., Kane, C.J. and Carroll, P.R. (2011) Treatment Trends for Stage I Renal Cell Carcinoma. Journal of Urology, 186, 394-399.

https://doi.org/10.1016/j.juro.2011.03.130

[15] Kane, C.J., Mallin, K., Ritchey, J., Cooperberg, M.R. and Carroll, P.R. (2008) Migration au stade du cancer des cellules rénales: analyse de la base nationale de données sur le cancer. Cancer, 113, 78-83. https://doi.org/10.1002/cncr.23518

[16] Schoots, I.G., Zaccai, K., Hunink, M.G. and Verhagen, P.C.M.S. (2017) Bosniak Classification for Complex Renal Cysts Reevaluated: A Systematic Review. Journal of Urology, 198, 12-21. https://doi.org/10.1016/j.juro.2016.09.160

[17] Jhaveri, K., Gupta, P., Elmi, A., et al. (2013) Cystic Renal Cell Carcinomas: Do They Develop, Metastasize or Reproduce? American Journal of Roentgenology, 201, W292-W296. https://doi.org/10.2214/AJR.12.9414

[18] Welch, H.G., Skinner, J.S., Schroeck, F.R., Zhou, W. and Black, W.C. (2018) Regional Variation in Computed Tomographic Imagery in the United States and Risk of Nephrectomy. JAMA Internal Medicine, 178, 221-227. https://doi.org/10.1001/jamainternmed.2017.7508

[19] Kashan, M., Ghanaat, M., Hötker, AM., et al. (2018) Cystic Renal Cell Carcinoma: A Report on the Results of Surgery and Active Surveillance in Patients Identified Retrospectively on Pretreatment Imaging. The Journal of Urology, 200, 275-282.

https://doi.org/10.1016/j.juro.2018.02.3087 


\section{Abbreviations}

MRI: Magnetic Resonance Imaging

CT: Computed Tomography 